\title{
Does transcutaneous electrical nerve stimulation (TENS) have a clinically relevant analgesic effect on different pain conditions? A literature review
}

\author{
Asami Naka (1), Mohammed Keilani (2), Stefan Loefler (1), Richard Crevenna (2)
}

(1) Ludwig Boltzmann Institute of Electrical Stimulation and Physical Rehabilitation, Wilhelminenspital, Vienna; (2) Department of Physical Medicine and Rehabilitation, Wilhelminenspital, Vienna; (3) Department of Physical Medicine and Rehabilitation, Medical University of Vienna, Austria

\begin{abstract}
Transcutaneous electric nerve stimulation (TENS) is a standard therapy used in different painful conditions such as low back pain, diabetic polyneuropathy or arthrosis. However, literature reviews focusing on the effects and the clinical implication of this method in various painful conditions are yet scarce. The purpose of this literature research was to determine, whether TENS provides an analgesic effect on common painful conditions in clinical practice. Literature research was performed using three data bases (Pubmed, Embase, Cochrane Database), focusing on papers published in the space of time from 2007 to 2012. Papers were evaluated from two reviewers independently concerning the clinical outcome, taking account for the level of external evidence according to the German Cochrane levels of evidence (Ia-IV). 133 papers of varying methodological quality dealing with different painful conditions were selected in total. A clinically relevant analgesic effect was described in 90 painful conditions (67\%). In 30 painful states (22\%), the outcome was inconclusive due to the study design. No significant analgesic effect of TENS was observed in 15 painful conditions $(11 \%)$. The vast majority of the papers were classified as Cochrane evidence level $\mathrm{Ib}(\mathrm{n}=64$; $48 \%)$, followed by level Ia $(n=23 ; 17 \%)$, level III $(n=18 ; 14 \%)$, level IV $(n=15 ; 11 \%)$, level $\mathrm{IIb}(\mathrm{n}=10 ; 8 \%)$ and level IIa $(\mathrm{n}=3 ; 2 \%)$. Most of the studies revealed an analgesic effect in various painful conditions, confirming the usefulness of TENS in clinical practice.

Key Words: Review, TENS, transcutaneous electric nerve stimulation, low frequency electrical stimulation, pain, analgesia, clinic
\end{abstract}

European Journal Translational Myology - Basic Applied Myology 2013; 23 (3): 95-103

Transcutaneous electric nerve stimulation (TENS) is a common therapeutic device used in different painful situations in the everyday clinical practice. It is noninvasive, inexpensive and reveals hardly any major side effects. TENS can be easily self-administered by the patients following a short training, and the intensity of electrical stimulation can be adjusted by the patients themselves according to need. Previous meta-analysis and reviews have reported about the effect of TENS in various painful conditions [1,3-6]. However, papers analyzed in meta-analysis or reviews revealed study designs of higher quality such as randomized controlled trials. Controlled studies without randomization, quasi-experimental studies or experts' opinion based on clinical observations were generally elided due to the poor study quality. Because of the limited or contradictory data, the effect of TENS was often evaluated as inconclusive. As stated by Sackett et al., the use of the best available scientific evidence from literature research solely is not sufficient, but requires the integration of individual clinical expertise [7]. Indeed in the daily clinics, the choice for the appropriate therapeutic modality is not based on the level of scientific evidence solely, but rather has to be customized according to the patients' need. The statement for inconclusiveness because of limited data or poor study design could distort the effectiveness of TENS in various painful conditions, and thus unethically deprive patients of effective treatment.

Therefore, the aim of this descriptive literature research was to provide an overview of papers including all kinds of study design dealing with the use of TENS in different painful situations. Clinical 
Table 1. Levels of external scientific evidence according to the German Cochrane Center.
Ia Systematic review based on randomized controlled trials with methodologically high quality
Ib Methodologically well designed randomized controlled trial
IIa Controlled study with high quality without randomization
IIb Study with a quasi-experimental design
III Non experimental study of high quality
IV Experts' options, descriptive studies such as case reports

effectiveness of TENS taking into account the levels of external scientific evidence according to Cochrane was evaluated.

\section{Materials and Methods}

For a structured literature search, three different electronic data bases (Pubmed, Embase, Cochrane Central Register of Controlled Trials) were used to screen for papers published from January 2007 to May 2012. "TENS", "transcutaneous electrical nerve stimulation" and "pain" were used as key words. Only articles in English or German were selected. AN and MK acted as reviewers and evaluated the clinical outcome (positive/negative/inconclusive), while RC supervised the work. Since the aim of the current literature research was focused on the analgesic effect of TENS in patients, studies from basic research, such as papers dealing with non-human models, were excluded. The remaining studies were further classified according to the scientific levels of evidence of the German Cochrane Center ([6]; see Table 1). Control group was defined as a group obtaining either shamTENS, where no electrical stimulation was given, or low threshold stimulation below the patients' detection threshold.

\section{Results and Discussion}

More than 800 hits were found in total, including painful conditions such as acute or chronic low back pain, osteoarthritis of the knee, rotator cuff tendinitis, cancer-related pain, postoperative pain, pain during child birth, painful diabetic neuropathy tension headache, trigeminal neuropathia or myofascial pain syndrome. Thereof, 133 papers were useful for further analysis. In two papers (Crawford-Faucher 2010, Dubinsky and Miyasaki 2010), two different painful conditions were described (positive outcome in painful diabetic neuropathy, negative outcome in chronic low back pain, respectively), hence resulting in a total of 135 outcomes. (see Table 2). The number of treated patients per study varied from eight to1466 (mean value $=141$, standard error of the mean $=27$ ).

An analgesic effect of TENS was reported in 90 out of 135 painful conditions in total ("positive"; 67\%). In 15 painful states (11\%), no alleviation of pain was observed ("negative"). The rest of the studies $(n=30$; $22 \%$ ) was inclusive, e. g. due to poor statistical quality, limited and/or contradictory data in studies with level
Ia, because of the missing statement about the outcome in case of reviews or experts' opinions, the use of other therapeutic interventions as control, or due to the study design (study protocol, methodological papers).

The vast majority of the papers were classified as evidence level $\mathrm{Ib}$ (randomized controlled trial), showing mostly a statistically significant analgesic effect of TENS. Second most common were level Ia studies (systematic review based on randomized controlled trials), consisting of mostly inconclusive studies, followed by papers from level III (nonexperimental studies, such descriptive case series) and level IV (experts' opinion based on the clinical experience, descriptive studies). Only few studies were graded as level IIa (experimental studies without randomization) or level IIb (studies of quasiexperimental design, such as case-control studies or cohort studies). See summary in Fig. 1.

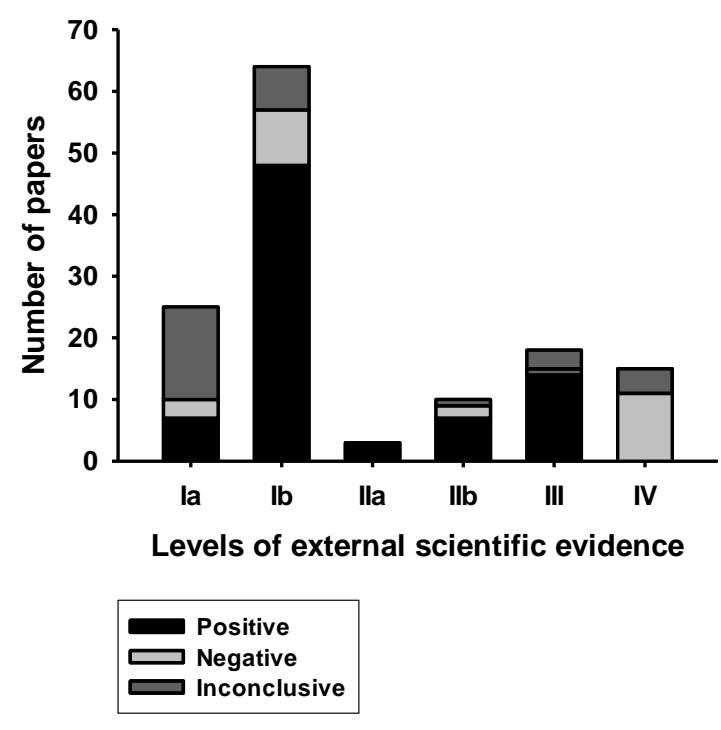

Fig 1. Studies dealing with the use of TENS in different painful conditions were classified according to the outcome and to the levels of evidence. Scientific levels of evidence according to the German Cochrane Center are plotted against the number of the papers. Studies were categorized according to the analgesic effect of TENS described in different gray scales 


\section{Analgesic effect of TENS in different painful conditions. A literature review}

European Journal Translational Myology - Basic Applied Myology 2013; 23 (3): 95-104

Many of the papers published between January 2007 and May 2012 were graded in higher levels of evidence. The majority of the studies showed an analgesic effect of TENS in various painful conditions, encouraging the continuing use of TENS in the everyday clinical practice. Nevertheless, taking into account the large amount of inconclusive studies graded in Cochrane level Ia, further randomized, double-blind, placebo-controlled studies revealing a statistically adequate number of patients are required to provide a better statement.

As mentioned above, good clinical practice requires the use of both best available scientic evidence and individual clinical expertise. Hence, low grading according to the Cochrane levels of scientific evidence does not automatically mean that these papers contain less clinically useful information. For example, the literature review from Pieber et al. (2010) contains much clinical details about the use of electrotherapy in painful diabetic peripheral neuropathy, considering the stimulation mode, stimulation intensity and frequency, time span of application, as well as the outcome, providing a good clinical overview. However, since it contains not only randomized controlled trials but also non-randomized controlled studies, uncontrolled studies or pilot studies, and because no statistical analysis was performed, it is graded as level IV, showing the difficulty of achieving both high scientific evidence and clinical relevance.

External scientific evidence allone can not replace the individual clinical expertise and vice versa. Although grading of papers in aspect of both scientific reliability and clinical usefulness is a challenging task, it is essential for the patients' sake and still remains to be optimized.

Table 2. Papers are graded according to the Cochrane levels of scientific evidence.

\begin{tabular}{|c|c|}
\hline Author & Reference \\
\hline \multicolumn{2}{|l|}{ Level Ia - positive outcome } \\
\hline $\begin{array}{l}\text { Bjordal JM, Johnson MI, Lopes-Martins RA, Bogen B, Chow R, } \\
\text { Ljunggren AE. }\end{array}$ & $\begin{array}{l}\text { Short-term efficacy of physical interventions in osteoarthritic knee pain. } \\
\text { A systematic review and meta-analysis of randomised placebo- } \\
\text { controlled trials. BMC Musculoskelet Disord 2007; 8:51. }\end{array}$ \\
\hline Claydon LS, Chesterton LS, Barlas P, Sim J. & $\begin{array}{l}\text { Dose-specific effects of transcutaneous electrical nerve stimulation } \\
\text { (TENS) on experimental pain: a systematic review. } \\
\text { Clin J Pain 2011; 7:635-47. }\end{array}$ \\
\hline Crawford-Faucher A. & $\begin{array}{l}\text { TENS helpful for management of diabetic neuropathy pain. } \\
\text { Am Fam Physician 2010; 82(8):991-2. } \\
\text { Positive outcome for painful diabetic neuropathy. }\end{array}$ \\
\hline Dowswell T, Bedwell C, Lavender T, Neilson JP. & $\begin{array}{l}\text { Transcutaneous electrical nerve stimulation (TENS) for pain relief in } \\
\text { labour. Cochrane Database Syst Rev 2009; 2:CD007214. }\end{array}$ \\
\hline Dubinsky RM, Miyasaki J. & $\begin{array}{l}\text { Assessment: efficacy of transcutaneous electric nerve stimulation in the } \\
\text { treatment of pain in neurologic disorders (an evidence-based review): } \\
\text { report of the Therapeutics and Technology Assessment Subcommittee } \\
\text { of the American Academy of Neurology. Neurology 2010; 74(2):173-6. } \\
\text { Positive outcome for painful diabetic neuropathy. }\end{array}$ \\
\hline Jin DM., Xu Y, Geng D-F., Yan T-B. & $\begin{array}{l}\text { Effect of transcutaneous electrical nerve stimulation on symptomatic } \\
\text { diabetic peripheral neuropathy: A meta-analysis of randomized } \\
\text { controlled trials. Diabetes Res Clin Pr 2010;89(1):10-5. }\end{array}$ \\
\hline Johnson M., Martinson M. & $\begin{array}{l}\text { Efficacy of electrical nerve stimulation for chronic musculoskeletal } \\
\text { pain: A meta-analysis of randomized controlled trials. Pain 2007; } \\
130(1-2): 157-165 \text {. }\end{array}$ \\
\hline \multicolumn{2}{|l|}{ Level Ia - negative outcome } \\
\hline Crawford-Faucher A. & $\begin{array}{l}\text { TENS helpful for management of diabetic neuropathy pain. Am Fam } \\
\text { Physician 2010; 82(8):9912. } \\
\text { Negative outcome for chronic low back pain. }\end{array}$ \\
\hline Dubinsky RM, Miyasaki J. & $\begin{array}{l}\text { Assessment: efficacy of transcutaneous electric nerve stimulation in the } \\
\text { treatment of pain in neurologic disorders (an evidence-based review): } \\
\text { report of the Therapeutics and Technology Assessment Subcommittee } \\
\text { of the American Academy of Neurology. Neurology_2010; 74(2):173-6. } \\
\text { Negative outcome for chronic low back pain. }\end{array}$ \\
\hline Mello LF, Nóbrega LF, Lemos A. & $\begin{array}{l}\text { Transcutaneous electrical stimulation for pain relief during labor: a } \\
\text { systematic review and meta-analysis. } \\
\text { Rev Bras Fisioter } 2011 ; 15(3): 175-84 \text {. }\end{array}$ \\
\hline \multicolumn{2}{|l|}{ Level Ia - inconclusive outcome } \\
\hline Bedwell C, Dowswell T, Neilson JP, Lavender T. & $\begin{array}{l}\text { The use of transcutaneous electrical nerve stimulation (TENS) for pain } \\
\text { relief in labour: a review of the evidence. Midwifery } 2011 ; 27(5): \mathrm{e} 141- \\
8 \text {. }\end{array}$ \\
\hline
\end{tabular}




\section{Analgesic effect of TENS in different painful conditions. A literature review}

European Journal Translational Myology - Basic Applied Myology 2013; 23 (3): 95-104

\begin{tabular}{|c|c|}
\hline Bennett MI, Hughes N, Johnson MI. & $\begin{array}{l}\text { Methodological quality in randomised controlled trials of } \\
\text { transcutaneous electric nerve stimulation for pain: low fidelity may } \\
\text { explain negative findings. Pain } 2011 ; 152(6): 1226-32 \text {. }\end{array}$ \\
\hline $\begin{array}{l}\text { Hurlow A, Bennett MI, Robb KA, Johnson MI, Simpson KH, } \\
\text { Oxberry SG. }\end{array}$ & $\begin{array}{l}\text { Transcutaneous electric nerve stimulation (TENS) for cancer pain in } \\
\text { adults. Cochrane Database Syst Rev 2012; 3:CD006276. }\end{array}$ \\
\hline Johnson MI, Bjordal JM. & $\begin{array}{l}\text { Transcutaneous electrical nerve stimulation for the management of } \\
\text { painful conditions: focus on neuropathic pain. } \\
\text { Expert Rev Neurother } 2011 ; 11(5): 735-53 \text {. }\end{array}$ \\
\hline Khadilkar A, Odebiyi DO, Brosseau L, Wells GA. & $\begin{array}{l}\text { Transcutaneous electrical nerve stimulation (TENS) versus placebo for } \\
\text { chronic low-back pain. Cochrane Database Syst Rev 2008; } \\
\text { 4:CD003008. }\end{array}$ \\
\hline $\begin{array}{l}\text { Kroeling P, Gross A, Goldsmith CH, Burnie SJ, Haines T, } \\
\text { Graham N, Brant A. }\end{array}$ & $\begin{array}{l}\text { Electrotherapy for neck pain. } \\
\text { Cochrane Back Group Cochrane Database of Syst Rev 2011; } 2 .\end{array}$ \\
\hline $\begin{array}{l}\text { Kroeling P, Gross A, Goldsmith CH, Burnie SJ, Haines T, } \\
\text { Graham N, Brant A. }\end{array}$ & $\begin{array}{l}\text { Electrotherapy for neck pain. } \\
\text { Cochrane Database Syst Rev 2009; 4:CD004251. }\end{array}$ \\
\hline Mello LF, Nóbrega LF, Lemos A. & $\begin{array}{l}\text { Transcutaneous electrical stimulation for pain relief during labor: a } \\
\text { systematic review and meta-analysis. Rev Bras Fisioter 2011; } \\
\text { 15(3):175-84. }\end{array}$ \\
\hline Mulvey MR, Bagnall AM, Johnson MI, Marchant PR. & $\begin{array}{l}\text { Transcutaneous electrical nerve stimulation (TENS) for phantom pain } \\
\text { and stump pain following amputation in adults. Cochrane Database Syst } \\
\text { Rev 2010; 5:CD007264. }\end{array}$ \\
\hline Nnoaham KE, Kumbang J. & $\begin{array}{l}\text { Transcutaneous electrical nerve stimulation (TENS) for chronic pain. } \\
\text { Cochrane Database Syst Rev 2008; 3:CD003222. }\end{array}$ \\
\hline Robb KA, Bennett MI, Johnson MI, Simpson KJ, Oxberry SG. & $\begin{array}{l}\text { Transcutaneous electric nerve stimulation (TENS) for cancer pain in } \\
\text { adults. Cochrane Database Syst Rev 2008; 3:CD006276. }\end{array}$ \\
\hline $\begin{array}{l}\text { Robb K, Oxberry SG, Bennett MI, Johnson MI, Simpson KH, } \\
\text { Searle RD. }\end{array}$ & $\begin{array}{l}\text { A cochrane systematic review of transcutaneous electrical nerve } \\
\text { stimulation for cancer pain. J Pain Symptom Manage 2009; 37(4):746- } \\
53 \text {. }\end{array}$ \\
\hline $\begin{array}{l}\text { Rutjes AW, Nüesch E, Sterchi R, Kalichman L, Hendriks E, Osiri } \\
\text { M, Brosseau L, Reichenbach S, Jüni P. }\end{array}$ & $\begin{array}{l}\text { Transcutaneous electrostimulation for osteoarthritis of the knee. } \\
\text { Cochrane Database Syst Rev 2009; 4:CD002823. }\end{array}$ \\
\hline $\begin{array}{l}\text { Van Middelkoop M., Rubinstein SM., Kuijpers T, Verhagen AP, } \\
\text { Ostelo R, Koes BW, Van Tulder MW. }\end{array}$ & $\begin{array}{l}\text { A systematic review on the effectiveness of physical and rehabilitation } \\
\text { interventions for chronic non-specific low back pain. Eur Spine J 2011; } \\
20(1): 19-39 \text {. }\end{array}$ \\
\hline Walsh DM, Howe TE, Johnson MI, Sluka KA. & $\begin{array}{l}\text { Transcutaneous electrical nerve stimulation for acute pain. } \\
\text { Cochrane Database Syst Rev 2009; 2:CD006142. }\end{array}$ \\
\hline \multicolumn{2}{|l|}{ Level $\mathrm{Ib}$ - positive outcome } \\
\hline Aghamohammadi A, Behmanesh F, Zafari M., Tofighi M. & $\begin{array}{l}\text { Effect of using transcutaneous electrical nerve stimulation (TENS) in } \\
\text { acupuncture points [Hegu (Li4) and Sanyinjiao (Sp6)] on duration of } \\
\text { the first stage of labor. Journal of Babol University of Medical Sciences } \\
2011 ; 13(2): 19-24 \text {. }\end{array}$ \\
\hline Akhmadeeva L, Veytsman B, Rayanova G, Setchenkova N. & $\begin{array}{l}\text { Dynamic TENS in management of low back pain: Cluster analysis for } \\
\text { efficiency prediction. Eur J Neurol 2010; conference: 14th Congress of } \\
\text { the European Federation of Neurological Societies, EFNS Geneva } \\
\text { Switzerland. Conference Start: } 20100925 \text { Conference End: } 20100928 \text {. } \\
\text { Conference Publication: (var.pagings). 17:584. }\end{array}$ \\
\hline $\begin{array}{l}\text { Amer-Cuenca JJ, Goicoechea C, Girona-Lopez A, Andreu-Plaza } \\
\text { JL, Palao-Roman R, Martinez-Santa G, Lison JF. }\end{array}$ & $\begin{array}{l}\text { Pain relief by applying transcutaneous electrical nerve stimulation } \\
\text { (TENS) during unsedated colonoscopy: A randomized double-blind } \\
\text { placebo-controlled trial. Eur J Pain } 2011 ; 15(1): 29-35 \text {. }\end{array}$ \\
\hline Bakhtiary AH, Asgari MR, Ebrahimian AA. & $\begin{array}{l}\text { Transcutaneous electrical nerve stimulations may reduce acute pain } \\
\text { during intravenous catheterization. Eur J Pain 2009. Conference: 6th } \\
\text { Congress of the European Federation of IASP Chapters: Pain in Europe } \\
\text { 6th, EFIC Lisbon Portugal. Conference Start: 20090909 Conference } \\
\text { End: 20090912. Conference Publication: (var.pagings). 13:S251-2. }\end{array}$ \\
\hline $\begin{array}{l}\text { Barbarisi M, Pace MC, Passavanti MB, Maisto M, Mazzariello L, } \\
\text { Pota V, Aurilio C. }\end{array}$ & $\begin{array}{l}\text { Pregabalin and transcutaneous electrical nerve stimulation for } \\
\text { postherpetic neuralgia treatment. Clin J Pain 2010;26(7):567-72. }\end{array}$ \\
\hline $\begin{array}{l}\text { Bennett MI, Johnson MI, Brown SR, Radford H, Brown JM, } \\
\text { Searle RD. }\end{array}$ & $\begin{array}{l}\text { Feasibility study of transcutaneous electrical nerve stimulation (TENS) } \\
\text { for cancer bone pain. J Pain 2010;11(4):351-9. }\end{array}$ \\
\hline $\begin{array}{l}\text { Boggio PS., Amancio EJ., Correa CF., Cecilio S, Valasek C, } \\
\text { Bajwa Z, Freedman SD, Pascual-Leone A, Edwards DJ, Fregni F. } \\
\end{array}$ & $\begin{array}{l}\text { Transcranial DC stimulation coupled with TENS for the treatment of } \\
\text { chronic pain: A preliminary study. Clin J Pain 2009; 25(8):691-5. }\end{array}$ \\
\hline Cetin N, Aytar A, Atalay A, Akman MN. & $\begin{array}{l}\text { Comparing hot pack, short-wave diathermy, ultrasound, and TENS on } \\
\text { isokinetic strength, pain, and functional status of women with } \\
\text { osteoarthritic knees: a singleblind, randomized, controlled trial. } \\
\text { Am J Phys Med Rehabil 2008; 87(6):443-51. }\end{array}$ \\
\hline Chandra A, Banavaliker JN, Das PK, Hasti S. & $\begin{array}{l}\text { Use of transcutaneous electrical nerve stimulation as an adjunctive to } \\
\text { epidural analgesia in the management of acute thoracotomy pain. } \\
\text { Indian J Anaesth } 2010 ; 54(2): 116-20 \text {. }\end{array}$ \\
\hline
\end{tabular}




\section{Analgesic effect of TENS in different painful conditions. A literature review}

European Journal Translational Myology - Basic Applied Myology 2013; 23 (3): 95-104

\begin{abstract}
Chao AS, Chao A, Wang TH, Chang YC, Peng HH, Chang SD, Chao A, Chang CJ, Lai CH, Wong AM.
\end{abstract}

Cheing GLY, Tsang HC, Lam CS, Chu PW, Yap J, Fung TY. Peres PA.

Coura LE, Manoel CH, Poffo R, Bedin A, Westphal GA

DeSantana JM, Santana-Filho VJ, Guerra DR, Sluka KA, Gurgel RQ, da Silva WM

Jr.

DeSantana JM, Sluka KA, Lauretti GR.

Pain relief by applying transcutaneous electrical nerve stimulation (TENS) on acupuncture points during the first stage of labor: a randomized double-blind placebocontrolled trial. Pain 2007; 127(3):214-20

A randomized controlled trial of auricular transcutaneous electrical nerve stimulation for managing posthysterectomy pain. Evid Based Complement Alternat Med 2011; 276769.

Short-term transcutaneous electrical nerve stimulation after cardiac surgery: effect on pain, pulmonary function and electrical muscle activity. Interact Cardiovasc Thorac Surg 2008; 7(4):539-43.

Randomised, controlled study of preoperative electroacupuncture for postoperative pain control after cardiac surgery. Acupunct Med 2011; 29 (1):16-20

Hypoalgesic effect of the transcutaneous electrical nerve stimulation following inguinal herniorrhaphy: a randomized, controlled trial. J Pain. 2008; 9(7):623-9.

High and low frequency TENS reduce postoperative pain intensity after laparoscopic tubal ligation: a randomized controlled trial. Clin J Pain 2009; 25(1):12-9.

Emmiler M, Solak O, Kocogullari C, Dundar U, Ayva E, Ela Y, Control of acute postoperative pain by transcutaneous electrical nerve Cekirdekci A, Kavuncu V. stimulation after open cardiac operations: a randomized placebocontrolled prospective study. Heart Surg Forum 2008;11(5):E300-3.

Eyigor C, Eyigor S, Kivilcim Korkmaz O. Are intra-articular corticosteroid injections better than conventional TENS in treatment of rotator cuff tendinitis in the short run? A randomized study. Eur J Phys Rehabil Med 2010; 46(3):315-24.

Ferreira FC, Issy AM, Sakata RK Assessing the effects of transcutaneous electrical nerve stimulation (TENS) in postthoracotomy analgesia. Rev Bras Anestesiol 2011; 61(5):561-7, 308-10.

Francis RP, Marchant PR, Johnson MI.

Comparison of post-treatment effects of conventional and acupuncturelike transcutaneous electrical nerve stimulation (TENS): A randomised placebo-controlled study using cold-induced pain and healthy human participants. Physiother Theory Pract. 2011; 27(8):578-85.

Freynet A, Falcoz PE

Is transcutaneous electrical nerve stimulation effective in relieving postoperative pain after thoracotomy? Interact Cardiovasc Thorac Surg 2010; 10(2):283-8.

Gemmell H, Hilland A. Immediate effect of electric point stimulation (TENS) in treating latent upper trapezius trigger points: a double blind randomised placebocontrolled trial. J Bodyw Mov Ther 2011; 15(3):348-54

Gschiel B, Kager H, Pipam W, Weichart K, Likar R.

[Analgesic efficacy of TENS therapy in patients with gonarthrosis. A prospective, randomised, placebo-controlled, double-blind study]. Schmerz 2010; 24(5):494-500.

Itoh K, Itoh S, Katsumi Y, Kitakoji H.

A pilot study on using acupuncture and transcutaneous electrical nerve stimulation to treat chronic non-specific low back pain. Complement Ther Clin Pract 2009; 15(1):22-5.

Kara B, Baskurt F, Acar S, Karadibak D, Ciftci L, Erbayraktar S, The effect of TENS on pain, function, depression, and analgesic Gokmen AN. consumption in the early postoperative period with spinal surgery patients. Turk Neurosurg. 2011;21(4):618-24

Lazarou L, Kitsios A, Lazarou I, Sikaras E, Trampas A.

Effects of intensity of Transcutaneous Electrical Nerve Stimulation (TENS) on pressure pain threshold and blood pressure in healthy humans: A randomized, double-blind, placebo-controlled trial. Clin J Pain 2009; 25(9):773-80.

\begin{tabular}{ll}
\hline Maayah M, Al-Jarrah M. & $\begin{array}{l}\text { Evaluation of Transcutaneous Electrical Nerve Stimulation as a } \\
\text { Treatment of Neck Pain due to Musculoskeletal Disorders. J Clin Med } \\
\text { Res 2010; 2(3):127-36. }\end{array}$ \\
\hline Man KM, Man SS, Shen JL, Law KS, Chen SL, Liaw WJ, Lee & $\begin{array}{l}\text { Transcutaneous electrical nerve stimulation on ST36 and SP6 acupoints } \\
\text { prevents hyperglycaemic response during anaesthesia: a randomised } \\
\text { CT, Lee YJ, Liao WL, Chang TM, Yu DX, Chen DC, Chen YH, }\end{array}$ \\
Chen WC, Chang SL. & $\begin{array}{l}\text { Hypoalgesia in response to transcutaneous electrical nerve stimulation } \\
\text { (TENS) depends on stimulation intensity. J Pain 2011; 12(8):929-35. }\end{array}$ \\
\hline Moran F, Leonard T, Hawthorne S, Hughes CM, McCrum- \\
Gardner E, Johnson MI, Rakel BA, Sluka KA, Walsh DM. & $\begin{array}{l}\text { Transcutaneous electrical nerve stimulation to treat vestibulodynia: A } \\
\text { randomized controlled trial. BJOG: An International Journal of } \\
\text { Obstetrics and Gynaecology 2008; 115(9):1165-70. }\end{array}$ \\
\hline Murina F, Bianco V, Radici G, Felice R, Di Martino M, Nicolini & $\begin{array}{l}\text { Adjusting pulse amplitude during transcutaneous electrical nerve } \\
\text { stimulation (TENS) application produces greater hypoalgesia. J Pain } \\
2011 ; 12(5): 581-90 .\end{array}$ \\
\hline Pantaleao MA, Laurino MF, Gallego NLG, Cabral CMN, Rakel \\
B, Vance C, Sluka KA, Walsh DM, Liebano RE. & $\begin{array}{l}\text { Immediate effects of transcutaneous electrical nerve stimulation and } \\
\text { focal knee joint cooling on quadriceps activation. Med Sci Sports Exerc } \\
2009 ; 41(6): 1175-81 .\end{array}$
\end{tabular}




\section{Analgesic effect of TENS in different painful conditions. A literature review}

European Journal Translational Myology - Basic Applied Myology 2013; 23 (3): 95-104

\begin{tabular}{|c|c|}
\hline $\begin{array}{l}\text { Platon B, Andréll P, Raner C, Rudolph M, Dvoretsky A, } \\
\text { Mannheimer C. }\end{array}$ & $\begin{array}{l}\text { High-frequency, high-intensity transcutaneous electrical nerve } \\
\text { stimulation as treatment of pain after surgical abortion. Pain. 2010; } \\
148(1): 114-9 \text {. }\end{array}$ \\
\hline $\begin{array}{l}\text { Ratajczak B, Hawrylak A, Demidaś A, Kuciel-Lewandowska J, } \\
\text { Boerner E. }\end{array}$ & $\begin{array}{l}\text { Effectiveness of diadynamic currents and transcutaneous electrical } \\
\text { nerve stimulation in disc disease lumbar part of spine. J Back } \\
\text { Musculoskelet Rehabil } 2011 ; 24(3): 155-9 \text {. }\end{array}$ \\
\hline Robb KA, Newham DJ, Williams JE. & $\begin{array}{l}\text { Transcutaneous electrical nerve stimulation vs. transcutaneous spinal } \\
\text { electroanalgesia for chronic pain associated with breast cancer } \\
\text { treatments. J Pain Symptom Manage 2007; 33(4):410-9. }\end{array}$ \\
\hline $\begin{array}{l}\text { Rodríguez-Fernández AL, Garrido-Santofimia } \\
\text { Rodríguez J, Fernándezde-Las-Peñas C. }\end{array}$ & $\begin{array}{l}\text { Effects of burst-type transcutaneous electrical nerve stimulation on } \\
\text { cervical range of motion and latent myofascial trigger point pain } \\
\text { sensitivity. Arch Phys Med Rehabil } 2011 ; 92(9): 1353-8 \text {. }\end{array}$ \\
\hline Saberkari MR, Nia KN. & $\begin{array}{l}\text { Transcutaneous electrical nerve stimulation (TENS) on postcesarean } \\
\text { section pain. Pain Practice 2009; conference: 5th World Congress - } \\
\text { World Institute of Pain. Conference Publication: (var.pagings). 9:150. }\end{array}$ \\
\hline Seenan C, Roche PA, Tan CW, Mercer T. & $\begin{array}{l}\text { Modification of experimental, lower limb ischemic pain with } \\
\text { transcutaneous electrical nerve stimulation. Clin J Pain 2012; } \\
\text { 28(8):693-9. }\end{array}$ \\
\hline Shimoji K, Takahashi N, Nishio Y, Koyanagi M, Aida S. & $\begin{array}{l}\text { Pain relief by transcutaneous electric nerve stimulation with } \\
\text { bidirectional modulated sine waves in patients with chronic back pain: } \\
\text { A randomized, double-blind, shamcontrolled study. Neuromodulation } \\
2007 ; 10(1): 42-51 \text {. }\end{array}$ \\
\hline Sikiru L, Shmaila H, Muhammed SA. & $\begin{array}{l}\text { Transcutaneous electrical nerve stimulation (TENS) in the symptomatic } \\
\text { management of chronic prostatitis/chronic pelvic pain syndrome: a } \\
\text { placebo-control randomized trial. Int Braz J Urol 2008; 34(6):708-13; } \\
\text { discussion } 714 \text {. }\end{array}$ \\
\hline Silva & $\begin{array}{l}\text { Analgesic Effect of Transcutaneous Electrical Nerve Stimulation After } \\
\text { Laparoscopic Cholecystectomy. Am J Phys Med Rehabil 2012; }\end{array}$ \\
\hline
\end{tabular}
91(8):652-7.

Solak O, Emmiler M, Ela Y, Dündar U, Koçoiullari CU, Eren N, Comparison of continuous and intermittent transcutaneous electrical Gökçe IY,

Cekirdekçi A, Kavuncu V. nerve stimulation in postoperative pain management after coronary artery bypass grafting: a randomized, placebo-controlled prospective study. Heart Surg Forum 2009; 12(5):E266-71.

Tsang HC, Lam CS, Chu PW, Yap J, Fung TY, Cheing GL.

A randomized controlled trial of auricular transcutaneous electrical nerve stimulation for managing posthysterectomy pain. Evid Based Complement Alternat Med 2011; 276769.

Unterrainer AF, Friedrich C, Krenn MH, Piotrowski WP, Postoperative and preincisional electrical nerve stimulation TENS Golaszewski SM, Hitzl W. reduce postoperative opioid requirement after major spinal surgery. $\mathrm{J}$ Neurosurg Anesthesiol 2010; 22(1):1-5.

Wu LL, Su CH, Liu CF. Effects of noninvasive electroacupuncture at hegu (LI4) and Sanyinjiao (SP6) acupoints on dysmenorrhea: A randomized controlled trial. J Altern Complement Med 2012; 18(2):137-42.

Yilmazer M, Kose S, Arioz DT, Koken G, Ozbulut O. Efficacy of transcutaneous electrical nerve stimulation for pain relief in women undergoing office endometrial biopsy. Arch Gynecol Obstet 2012; 285(4):1059-64.

Level Ib - negative outcome

Binder P, Gustafsson A, Uvnas-Moberg K, Nissen E. Hi-TENS combined with PCA-morphine as post caesarean pain relief. Midwifery 2011; 27(4): 547-52.

Francis RP, Marchant P, Johnson MI.

Conventional versus acupuncture-like transcutaneous electrical nerve stimulation on cold-induced pain in healthy human participants: effects during stimulation. Clin Physiol Funct Imaging 2011; 31(5):363-70.

Gossrau G, Wähner M, Kuschke M, Konrad B, Reichmann H, Microcurrent transcutaneous electric nerve stimulation in painful Wiedemann B, Sabatowski R. diabetic neuropathy: a randomized placebo-controlled study. Pain Med 2011; 12(6):953-60.

Navez ML, Buchmuller-Cordier A, Presles E, Techer J, El Asri G, Evaluation of transcutaneous neurostimulation on chronic lumbalgia or Laurent B. lumboradiculalgia for patients treated in French pain centres. European Journal of Neurology 2009; conference: 13th Congress of the EFNS Florence Italy. Conference Start: 20090912 Conference End: 20090915. Conference Publication: (var.pagings) 16(S3):72.

Oosterhof J, Wilder-Smith OH, Oostendorp RA, Crul BJ. Different mechanisms for the short-term effects of real versus sham Transcutaneous Electrical Nerve Stimulation (TENS) in patients with chronic pain: A pilot study. J Pain Palliat Care Pharmacother 2012; 26(1):5-12

Oosterhof J, Wilder-Smith OH, de Boo T, Oostendorp RA, Crul The long-term outcome of Transcutaneous Electrical Nerve Stimulation BJ. in the treatment for patients with chronic pain: A randomized, placebocontrolled trial. Pain Pract. 2012; 12(7):513-22. 


\section{Analgesic effect of TENS in different painful conditions. A literature review}

European Journal Translational Myology - Basic Applied Myology 2013; 23 (3): 95-104

Short-term effects of Transcutaneous Electrical Nerve Stimulation (TENS) and exercise on knee osteoarthritis (OA). Rheumatology 2010; conference: Rheumatology 2010 - British Society for Rheumatology, BSR and British Health Professionals in Rheumatology, BHPR Annual Meeting 2010. Birmingham United Kingdom. Conference Start: 20100420 Conference End: 20100423. Conference Publication: (var.pagings) 49:i33-i34.

Pourmomeny AA, Amini M, Safaei H, Hassanzadeh A.

The effect of electroanalgsia on pain relief in patient with diabetic neuropathy type II. Iranian Journal of Endocrinology and Metabolism 2009: 11(4)363-369+472.

Sahin N, Albayrak I, Ugurlu H.

Effect of different transcutaneous electrical stimulation modalities on cervical myofascial pain syndrome. J Musculoskelet Pain 2011: 19(1)18-23.

Level $\mathrm{Ib}$ - inconclusive outcome

Barker KL, Elliott CJ, Sackley CM, Fairbank JC.

Treatment of chronic back pain by sensory discrimination training. A Phase I RCT of a novel device (FairMed) vs. TENS. BMC Musculoskelet Disord 2008; 9:97.

Beckwée D, De Hertogh W, Lievens P, Bautmans I, Vaes P.

Effect of TENS on pain in relation to central sensitization in patients with osteoarthritis of the knee: study protocol of a randomized controlled trial. Trials 2012; 13:21.

Buchmuller A, Navez M, Milletre-Bernardin M, Pouplin S, Value of TENS for relief of chronic low back pain with or without Presles E, Lantéri-Minet M, Tardy B, Laurent B, Camdessanché radicular pain. Eur J Pain 2011; 16(5):656-65.

JP

Chesterton LS, van der Windt DA, Sim J, Lewis M, Mallen CD,

Mason EE, Warlow C, Vohora K, Hay EM.

Transcutaneous electrical nerve stimulation for the management of tennis elbow: a pragmatic randomized controlled trial: the TATE trial (ISRCTN 87141084)

BMC Musculoskelet Disord 2009; 10:156.

Dias M, Carneiro NM, Guerra LA, Velarde GC, de Souza PA, da Effects of electroacupuncture on local anaesthesia for inguinal hernia Silva LL, de Abreu e Souza RR, Nolasco R, Olej B. repair: a randomised placebo-controlled trial. Acupunct Med 2010. 28(2)65-70.

Léonard G, Goffaux P, Marchand S. Deciphering the role of endogenous opioids in high-frequency TENS using low and high doses of naloxone. Pain 2010; 151(1):215-9.

Léonard G, Cloutier C, Marchand S

Reduced analgesic effect of acupuncture-like TENS but not conventional TENS in opioid-treated patients. J Pain 2011; 12(2):21321

Level IIa - positive outcome

Chen CC, Johnson MI

Differential frequency effects of strong nonpainful transcutaneous electrical nerve stimulation on experimentally induced ischemic pain in healthy human participants. Clin J Pain, 2011; 27(5):434-41.

Kofotolis ND, Vlachopoulos SP, Kellis E. Sequentially allocated clinical trial of rhythmic stabilization exercises and TENS in women with chronic low back pain. Clin Rehabil 2008; 22(2):99-111.

Peng T, Li XT, Zhou SF, Xiong Y, Kang Y, Cheng HD. $\quad$ Transcutaneous electrical nerve stimulation on acupoints relieves labor pain: a nonrandomized controlled study. Chin J Integr Med 2010; 16(3):234-8.

Level Ilb - positive outcome

Buchmuller-Cordier A, Navez M, Presles E, Venet E, Dupont O, Laurent B.

First national survey of drug-free techniques used in French pain centers for the management of chronic pain in adults. <Premiere enquete nationale sur les techniques non medicamenteuses.> Douleurs 2008 9(6):315-19.

Chen CC, Johnson MI. An investigation into the hypoalgesic effects of high- and lowfrequency transcutaneous electrical nerve stimulation (TENS) on experimentally-induced blunt pressure pain in healthy human participants. J Pain 2010; 11(1):53-61.

Chen C, Johnson MI.

A comparison of transcutaneous electrical nerve stimulation (TENS) at 3 and 80 pulses per second on cold-pressor pain in healthy human participants. Clin Physiol Funct Imaging 2010; 30(4):260-8.

Escortell-Mayor E, Riesgo-Fuertes R, Garrido-Elustondo S, Primary care randomized clinical trial: manual therapy effectiveness in Álvarez E.

Löfgren M, Norrbrink C. comparison with TENS in patients with neck pain. Man Ther 2011; 16(1):66-73

Pain relief in women with fibromyalgia: a cross-over study of superficial warmth stimulation and transcutaneous electrical nerve stimulation. J Rehabil Med 2009; 41(7):557-62.

Miller L, Mattison P, Paul L, Wood L. The effects of transcutaneous electrical nerve stimulation (TENS) on spasticity in multiple sclerosis. Mult Scler 2007; 13(4):527-33. 


\section{Analgesic effect of TENS in different painful conditions. A literature review}

European Journal Translational Myology - Basic Applied Myology 2013; 23 (3): 95-104

\begin{tabular}{ll}
\hline Ward A.R., Lucas-Toumbourou S., McCarthy B. & $\begin{array}{l}\text { A comparison of the analgesic efficacy of medium-frequency } \\
\text { alternating current and TENS. Physiotherapy 2009; 95(4):280-8. }\end{array}$ \\
\hline Level Ilb - negative outcome & $\begin{array}{l}\text { A comparison of the hypoalgesic effects of transcutaneous electrical } \\
\text { nerve stimulation (TENS) and non-invasive interactive neurostimulation } \\
\text { (InterX(®) ) on experimentally induced blunt pressure pain using } \\
\text { healthy human volunteers. }\end{array}$ \\
\hline Biggs N, Walsh DM, Johnson MI. & $\begin{array}{l}\text { An investigation into the effect of electrode placement of } \\
\text { transcutaneous electrical } \\
\text { nerve stimulation (TENS) on experimentally induced ischemic pain in } \\
\text { healthy human } \\
\text { participants. } \\
\text { Clinical J Pain 2007; 23(9):735-73. }\end{array}$ \\
\hline
\end{tabular}

Level IIb - inconclusive outcome

Turna A, Pekcolaklar A, Metin M, Sayar A, Solak O, Gurses A. Transcutaneous electric nerve stimulation for the treatment of postthoracotomy pain: A randomized prospective study. Thorac Cardiov Surg 2007; 55 (3)182-5.

Level III - positive outcome

Bernhard MK, Himpel M, Syrbe S, Mirjam L, Merkenschlager A.

TENS therapy in tension headaches-little helps much?!.

Neuropediatrics 2011; conference: 37th Annual Meeting of the Society of Neuropediatrics Garmisch-Partenkirchen Germany. Conference Start: 20110407 Conference End: 20110410. Conference Publication: (var.pagings) 42 .

Buonocore M, Camuzzini N

Increase of the heat pain threshold during and after high-frequency transcutaneous peripheral nerve stimulation in a group of nor.mal subjects. Europa Medicophysica 2007; 43(2)155-60.

Chen CC, Tabasam G, Johnson MI

Does the pulse frequency of transcutaneous electrical nerve stimulation (TENS) influence hypoalgesia?. A systematic review of studies using experimental pain and healthy human participants. Physiotherapy 2008 ; 94(1):11-20)

Dionisi B, Senatori R.

Effect of transcutaneous electrical nerve stimulation on the postpartum dyspareunia treatment. J Obstet Gynaecol Res 2011; 37(7):750-3.

Fatemeh VR, Zahra S

thoracic-lumbo sacral vertebras on active phase of labor.

J Psychosom Obst Gyn 2010; conference: Abstract of $15^{\text {th }}$ Internationa

Congress of ISPOG Venezia Italy. Conference Start: 20101028

Conference End: 20101030. Conference Publication: (var.pagings) $31: 125$

Grazio S, Markuiincic B, Muraja S, Grubisic F, Nemcic T, Efficacy of low-level laser therapy and transcutaneous electrical nerve Matijevic V, Skala H, Kasun B, Susak V, Koprivnjak V, Trgovec stimulation (TENS) therapy on level of pain, global assessment of the disease and function in chronic low-back pain. Clin Exp Rheumatol 2009; conference: 13th Mediterranean Congress of Rheumatology Cavtat Croatia. Conference Start: 20091118 Conference End 20091121. Conference Publication: (var.pagings) 27 (5):725-26.

Kavak S, Tulgar M, Anlar O.

Effects of transcutaneous electrical nerve stimulation on motor and sensorial nerves for diabetic polyneuropathy patients by use of electromyography. Cell Membranes and Free Radical Research 2010; 2 (3):112-6.

Moharic M, Burger H

Effect of transcutaneous electrical nerve stimulation on sensation thresholds in patients with painful diabetic neuropathy: an observational study. Int J Rehabil Res 2010; 33(3):211-7.

Norrbrink C.

Transcutaneous electrical nerve stimulation for treatment of spinal cord injury neuropathic pain. J Rehabil Res Dev 2009; 46(1):85-93.

Rajpurohit B, Khatri SM, Metgud D, Bagewadi A Effectiveness of transcutaneous electrical nerve stimulation and microcurrent electrical nerve stimulation in bruxism associated with masticatory muscle pain - a comparative study. Indian J Dent Res 2010; 21(1):104-6.

\begin{tabular}{ll}
\hline Schiotz HA, Jettestad M, Al-Heeti D. & $\begin{array}{l}\text { Treatment of dysmenorrhoea with a new TENS device (OVA). J Obst } \\
\text { Gyn 2007; 27(7):726-8. }\end{array}$ \\
\hline Singla S, Prabhakar V, Singla RK. & $\begin{array}{l}\text { Role of transcutaneous electric nerve stimulation in the management of } \\
\text { trigeminal neuralgia. J Neurosci Rural Pract 2011; 2(2)150-2. }\end{array}$ \\
\hline Tugay N, Akbayak T, Karakaya IC, Kocaacar O, Tugay U, & $\begin{array}{l}\text { Effectiveness of transcutaneous electrical nerve stimulation and } \\
\text { interferential current in primary dysmenorrhea. Pain Med 2007; } \\
\text { Karakaya MG, Demirturk F. }\end{array}$
\end{tabular}




\section{Analgesic effect of TENS in different painful conditions. A literature review}

European Journal Translational Myology - Basic Applied Myology 2013; 23 (3): 95-104

\section{Yameen F, Shahbaz NN, Hasan Y, Fauz R, Abdullah M.}

Level III - negative outcome

Engholm G, Leffler AS

Efficacy of transcutaneous electrical nerve stimulation and its different modes in patients with trigeminal neuralgia. J Pak Med Assoc 2011; 61(5):437-9.

Influence of pain reduction by Transcutaneous Electrical Nerve Stimulation (TENS) on sensory dysfunction in patients with painful peripheral nerve injury. Eur J Pain 2009; conference: 6th Congress of the European Federation of IASP Chapters: Pain in Europe 6th, EFIC Lisbon Portugal. Conference Start: 20090909 Conference End: 20090912. Conference Publication: (var.pagings) 13:S166

Level III - inconclusive outcome

Mysliwiec A, Saulicz E, Kuszewski M, Kokosz M., Wolny T.

Assessment of the influence of Saunders traction and transcutaneous electrical nerve stimulation on hand grip force in patients with neck pain. Ortop Traumatol Rehabil. 13(1):37-44

A comparison of high- versus low-intensity, high-frequency transcutaneous electric nerve stimulation for painful postpartum uterine contractions. Acta Obstet Gynecol Scand 2007; 86(3):310-4.

Olsen MF, Elden H, Janson ED, Lilja H, Stener-Victorin E.

The effect of TENS on selected symptoms in the management of

Tella BA, Unubum EV, Danesi MA

patients with chronic tension type headache: a preliminary study. Nig Q J Hosp Med 2008; 18(1)25-9.

Level IV - positive outcome

Binder A, Baron R.

Utility of transcutaneous electrical nerve stimulation in neurologic pain disorders. Comment on Neurology 2010; 74(2):173-6. Neurology 2010;74(2):104-5.

Bjordal JM.

Time for a paradiom shift in pain treatment: reassessing transcutaneous electrical nerve stimulation (TENS). Comment on Pain 2011; 152(6):1226-32. Pain 2011; 152(6):1213-4.

DeSantana JM, Walsh DM, Vance C, Rakel BA, Sluka KA.

Effectiveness of transcutaneous electrical nerve stimulation for treatment of hyperalgesia and pain. Curr Rheumatol Rep 2008; 10(6):492-9.

Meissner W.

The role of acupuncture and transcutaneous-electrical nerve stimulation for postoperative pain control. Curr Opin Anaesthesiol 2009; 22(5):6236.

Pieber K, Herceg M, Paternostro-Sluga T.

Plastaras CT, Schran S, Kim N, Sorosky S, Darr D, Chen MS Lansky R.

Electrotherapy for the treatment of painful diabetic peripheral neuropathy: a review. J Rehabil Med 2010; 42(4):289-95.

Complementary and alternative treatment for neck pain: chiropractic, acupuncture, TENS, massage, yoga, Tai Chi, and Feldenkrais. Phys Med Rehabil Clin N Am 2011; 22(3):521-37, ix

Rode G, Vallar G, Revol P, Tilikete C, Jacquin-Courtois S, Facial macrosomatognosia and pain in a case of Wallenberg's Rossetti Y, Farne A

\begin{tabular}{|c|c|}
\hline Rojahn R. & $\begin{array}{l}\text { Transcutaneous electrostimulation for osteoarthritis of the knee. } \\
\text { J Pain Manag 2011; 4(4):447-9. }\end{array}$ \\
\hline Searle RD, Bennett MI, Johnson MI, Callin S, Radford H. & $\begin{array}{l}\text { Transcutaneous electrical nerve stimulation (TENS) for cancer bone } \\
\text { pain. J Pain Symptom Manage 2009; 37(3):424-8. }\end{array}$ \\
\hline Tashani O, Johnson M. & $\begin{array}{l}\text { Transcutaneous electrical nerve stimulation (TENS) A possible aid for } \\
\text { pain relief in developing countries? Libyan J Med } 2009 ; 4(2): 62-5 \text {. }\end{array}$ \\
\hline Ying KN, While A. & $\begin{array}{l}\text { Pain relief in osteoarthritis and rheumatoid arthritis: TENS. } \\
\text { Br J Community Nursing 2007; 12(8):364-71. }\end{array}$ \\
\hline \multicolumn{2}{|l|}{ Level IV - inconclusive outcome } \\
\hline Jayasekara R. & $\begin{array}{l}\text { Transcutaneous electrical nerve stimulation for acute pain. } \\
\text { J Pain Manag 2011; 4(4):437-8. }\end{array}$ \\
\hline $\begin{array}{l}\text { Peter WFH, Jansen MJ, Hurkmans EJ, Bloo H, Dekker-Bakker } \\
\text { LMMCJ, Dilling RG, Hilberdink WKHA, Kersten-Smit C, de } \\
\text { Rooij M, Veenhof C, Vermeulen HM, de Vos I, Schoones JW, } \\
\text { Vliet Vlieland TPM. }\end{array}$ & $\begin{array}{l}\text { Physiotherapy in hip and knee osteoarthritis: Development of a practice } \\
\text { guideline concerning initial assessment, treatment and evaluation. } \\
\text { Acta Reumatol Port } 2011 ; 36(3): 268-81 \text {. }\end{array}$ \\
\hline Scudds RJ, Scudds RA, Baxter GD, McDonough SM, Walsh DM. & $\begin{array}{l}\text { Transcutaneous electrical nerve stimulation for the treatment of pain in } \\
\text { physiotherapy practices in Hong Kong and the United Kingdom - a } \\
\text { survey of usage and perceived effectiveness compared with other pain } \\
\text { relieving modalities. Hong Kong Physiotherapy Journal } 2009 ; 27: 11-20 \text {. }\end{array}$ \\
\hline Van Epps S, Zempsky W, Schechter N, Pescatello LS, Lerer T. & $\begin{array}{l}\text { The effects of a two-week trial of transcutaneous electrical nerve } \\
\text { stimulation for pediatric chronic back pain. J Pain Symptom Manage } \\
2007 ; 34(2): 115-7 \text {. }\end{array}$ \\
\hline
\end{tabular}




\section{Analgesic effect of TENS in different painful conditions. A literature review}

European Journal Translational Myology - Basic Applied Myology 2013; 23 (3): 95-104

\section{Corresponding Author}

Univ.-Prof. Dr. Richard Crevenna, Medical University of Vienna, Waehringer Guertel 18-20, AT-1090 Vienna, Austria

Phone +43 40400 4330; Fax +43 404005281

e-mail: richard.crevenna@meduniwien.ac.at

\section{References}

[1] Bedwell C, Dowswell T, Nellosn JP, Lavender T (2011). The use of transcutaneous electrical nerve stimulation (TENS) for pain relief in labour: a review of the evidence. Midwifery 27(5):e141-8. Epub 2010 Feb 18.

[2] Höltl W, Loidl W, Rauchenwald M, Müller M, Klimpfinger M, Schratter-Sehn A, Brössner C (1992). Prostate Biopsy Guidelines of the AUO Study Group Urologic Oncology of the Austrian Society of Urologists and Andrologists. Levels of evidence. AHCPR publication 92-0032: 100-107.

[3] Johnson M, Martinson M (2007). Efficacy of electrical nerve stimulation for chronic musculoskeletal pain: A meta-analysis of randomized controlled trials. Pain. 130(1-2):157165.

[4] Kroeling P, Gross A, Goldsmith CH, Burnie SJ, Haines T, Graham N, Brant A (2009) Electrotherapy for neck pain. Cochrane Database Syst Rev. Oct 7;(4):CD004251.

[5] Robb KA, Bennett MI, Johnson MI, Simpson KJ, Oxberry SG (2008) Transcutaneous electric nerve stimulation (TENS) for cancer pain in adults. Cochrane Database Syst Rev. Jul 16;(3):CD006276.

[6] Rutjes AW, Nüesch E, Sterchi R, Kalichman L, Hendriks E, Osiri M, Brosseau L, Reichenbach S, Jüni P (2009). Transcutaneous electrostimulation for osteoarthritis of the knee. Cochrane Database Syst Rev. Oct 7;(4):CD002823.

[7] Sackett DL, Rosenberg WM, Gray JA, Haynes RB, Richardson WS (1996). Evidence based medicine: what it is and what it isn't. BMJ 312(7023):71-2. 